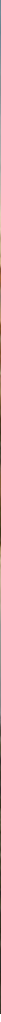

\title{
Frühe Jahre der Reformation in Torgau bis 1529
}

\author{
Jürgen Herzog
}

Die Stadt Torgau war in besonderem Maße seit den letzten 15 Jahren des 15. Jahrhunderts bis zur Mitte des 16. Jahrhunderts vom Dualismus zwischen Landesherrschaft und landesstädtischer Bürgergemeinde geprägt. ${ }^{1}$ Im Ergebnis der Leipziger Landesteilung Sachsens 1485 gingen für das nunmehrige Kurfürstentum bisherige Standorte der Herrschaftsausübung, Dresden, Leipzig und Meißen, verloren. Torgau wurde Hauptsitz der Kurfürstlichen Kanzlei und der landesherrschaftlichen Administration. ${ }^{2}$ Damit war die häufige Anwesenheit des kurfürstlichen Hoflagers und des Landesherren in der Stadt verbunden. Das Hoflager Friedrich des Weisen (1463-1525) war von 1486 bis
1516 jährlich durchschnittlich etwa 23 Wochen in Torgau anwesend, danach hielten sich der Kurfürst und sein Hof vorwiegend auf dem nahegelegenen Jagdschloss, der Lochau, auf. ${ }^{3}$ Kurfürst Johann (1468-1532) und sein Sohn Kurfürst Johann Friedrich (1503-1554) residierten nur noch in der Hauptresidenz Torgau und in der Nebenresidenz Weimar. ${ }^{4}$

Die Bürgerstadt Torgau verfügte seit dem 15. Jahrhundert über alle einer sächsischen Landesstadt möglichen Freiheiten, so die der Erbund Obergerichtsbarkeit und der Landtagsfähigkeit. Im Jahr 1524 gab es 480 steuernde bürgerliche Hausstellen und Torgau hatte einschließlich der Eigentümer von Freihäusern,

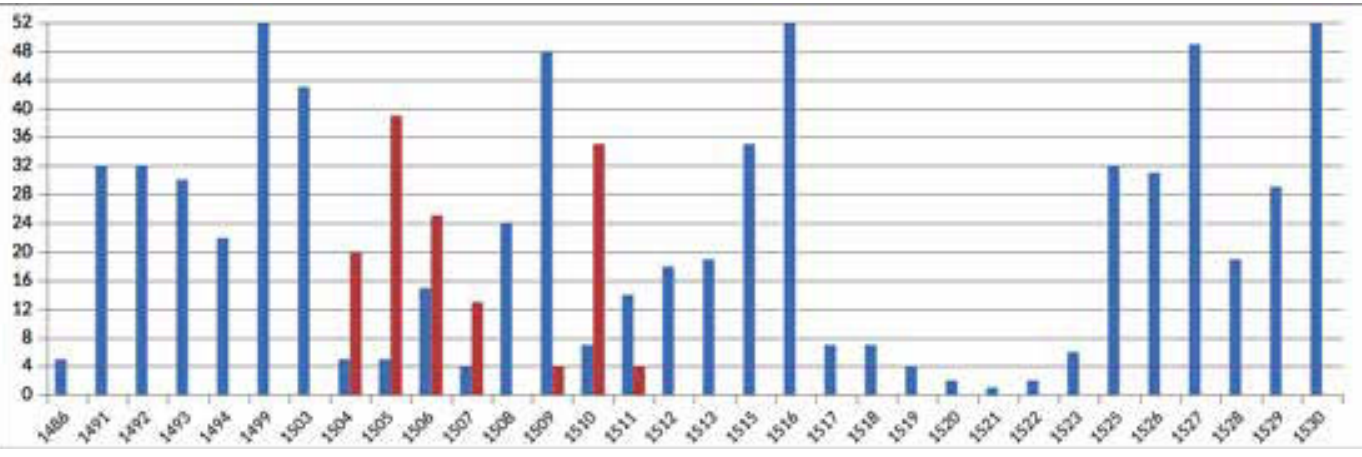

Schloss Hartenfels in Torgau, Ölgemälde von Lucas Cranach d. J., um 1544

(c) Wikimedia

1 Karlhein Blaschke, Die geschichtliche Entwicklung der Stadt Torgau ..., in: Peter Findeisen und Heinrich Magirius, Die Denkmale der Stadt Torgau, Leipzig 1976, S. 27.

2 Ingetraud Ludolphy, Friedrich der Weise, Göttingen 1984, S. 292

3 Jürgen Herzog, Fürstlicher Hof und Stadt Torgau während der Regierungszeit Friedrich des Weisen, in: Kurfürst Friedrich der Weise von Sachsen, Quellen und Forschungen zur sächsischen Geschichte, Bd. 40, Hrsg. Armin Kohnle und Uwe Schirmer, Stuttgart 2015, S. 294.

Hoflager der Kurfürsten und des Kurprinzen Johann Friedrich in Torgau, Angabe in Wochen 1486-1530, bearbeitet von Jürgen Herzog

๔ Jürgen Herzog 
4 Jürgen Herzog, Vorreformatorische Kirche und Reformation in Torgau, Schriften des Torgauer Geschichtsvereins, Bd. 10, Beucha/Markkleeberg 2016, S. 230.

5 Herzog, Fürstlicher Hof (wie Anm. 3), S. 321.

6 Herzog, Kirche und Reformation (wie Anm. 4), S. 74, S. 99 ff. und 159

7 Johann Burchard Mencke, Scriptores rerum Germanicarum praecique Saxonicarum, Teil 2, Lipsiae 1728, S. 583; Stadtarchiv Torgau (im Folgenden: StAT), H 49, Wilhelm Krudthoff, Historischer Stromateus torgauischer Alterthümer, S. 103.

8 StAT H 39, Torgauer Chronik, S. 23.

9 Johann Theodor Lingke, Herrn D. Martin Luthers Geschäfte und Andenken in Torgau, Torgau 1764, S. 2; Georg Buchwald, Luther-Kalendarium, in: Schriften des Vereins für Reformationsgeschichte, Jahrgang 47, H. 2, Leipzig 1929, S. 12

10 Krudthoff, Stromateus (wie Anm. 7), S. 106.

11 Martin Luthers Werke, Weimar 1883 ff, Briefe, Band 2, S. 282, Nr. 384 und S. 283, Nr. 385 (im Folgenden: WA, Briefe WA BR und Tischreden WA TR).

Ansicht von Torgau, Ausschnitt mit Kirchgang, Zeichnung, Wilhelm Dilich, 1629 aus: Publikationen des AltertumsVereins zu Torgau III, Torgau 1889, Beilage zu S. 18 der Geistlichkeit und besitzloser Pfahlbürger, jedoch ohne das nichtbürgerliche Hofpersonal, etwa 3.300 Einwohner. $^{5}$

Eine umfassende Ausweitung der Frömmigkeitspraxis in den Jahrzehnten vor und nach 1500 war insbesondere mit der Person Kurfürst Friedrich des Weisen verbunden. In diesem $\mathrm{Zu}$ sammenhang stand die Hauptkirche der Stadt Torgau, die Parochialkirche Unser lieben Frauen, im Zentrum landesherrschaftlicher Stiftungstätigkeit. Die Landesherrschaft verfügte nicht nur über die Patronatsrechte für Pfarrei und Kirche, sondern hatte auch für sieben von 17 Altarstiftungen das Patronat inne. Spätestens mit der Beisetzung der jung verstorbenen Herzogin Sophie von Sachsen (1481-1503), der ersten Frau Herzog Johanns, und der Errichtung eines erhöhten und überdachten Kirchgangs vom Schloss zur Kirche erreichte sie den Status einer Hofkirche.

Im Gegensatz dazu war die Marktkirche St. Nikolai die eigentliche Bürgerkirche. Dieser noch spätromanisch geprägte Bau, der als Gründungskirche der planmäßig angelegten Bürgerstadt westlich des Marktes anzusehen ist, verfügte über acht Altarstiftungen, von denen nur eine landesherrschaftlich gewesen ist. Hier hatte der Torgauer Rat bei Mitspracherechten geistlicher Bruderschaften die Patronatsrechte inne. Um 1500 wurden in den zehn Kirchen und Kapellen in und vor der Stadt jährlich mindestens 8.000 Messen abgehalten. ${ }^{6}$

Unter den Bedingungen fürstlicher Prägung und persönlicher Präsenz war die reformatorische Bewegung in der Stadt in besonderem
Maße von der Persönlichkeit Friedrich des Weisen und seiner zwar gewährenden, insgesamt aber doch altgläubig bestimmten Haltung beeinflusst, die den Handlungsspielraum des Torgauer Rates bis zum Lebensende des Kurfürsten im Jahr 1525 begrenzt hat.

Die ersten reformatorisch geprägten Ereignisse sind nur chronikalisch überliefert und deshalb mit Unsicherheiten verbunden. Schon 1518 sollen der seit 1514 als Prediger in Torgau nachweisbare Valentin Tham (um 1490-1548) und der Mönch Franziscus Mechler aus dem Torgauer Franziskanerkloster gegen die päpstliche Religion und den Ablass gepredigt haben. 1519 war „[...] der gebrauch lateinisch zu tauffen abgeschafft, vnd die Hanß Entzwirtin vnd des Hanß Tünchers Tochter in der Niklas Kirche, zu erst deutsch seyn geteuffet worden“. ${ }^{8}$ Martin Luther predigte erstmals im Oktober 1519 hier und erhielt je ein Stübchen rheinischen Wein und Met für fünf Groschen und drei Pfennig. ${ }^{9}$ Valtentin Tham zugeschrieben ist am 5. August 1520 in der Nikolaikirche „[...] die erste Evangelische Predigt, vom ungerechten Haußhalter abgeleget worden [...] solcher Teyl aber ist denen päpstlern Einrecht gegebener Abschied gewesen, thut Rechnung von euern Haußhalten, denn ihr könnet hinfort nicht mehr Haußhalter seyn." 10

Als im Januar 1521 Martin Luther mit dem Bann bedroht und kirchenrechtlich zum Ketzer erklärt worden war, wurde auch in Torgau wie anderswo die heimlich angeschlagene päpstliche Bulle gleich darauf zerrissen. ${ }^{11}$ In diesem Jahr 1521 begann sich Luthers Lehrmeinung,

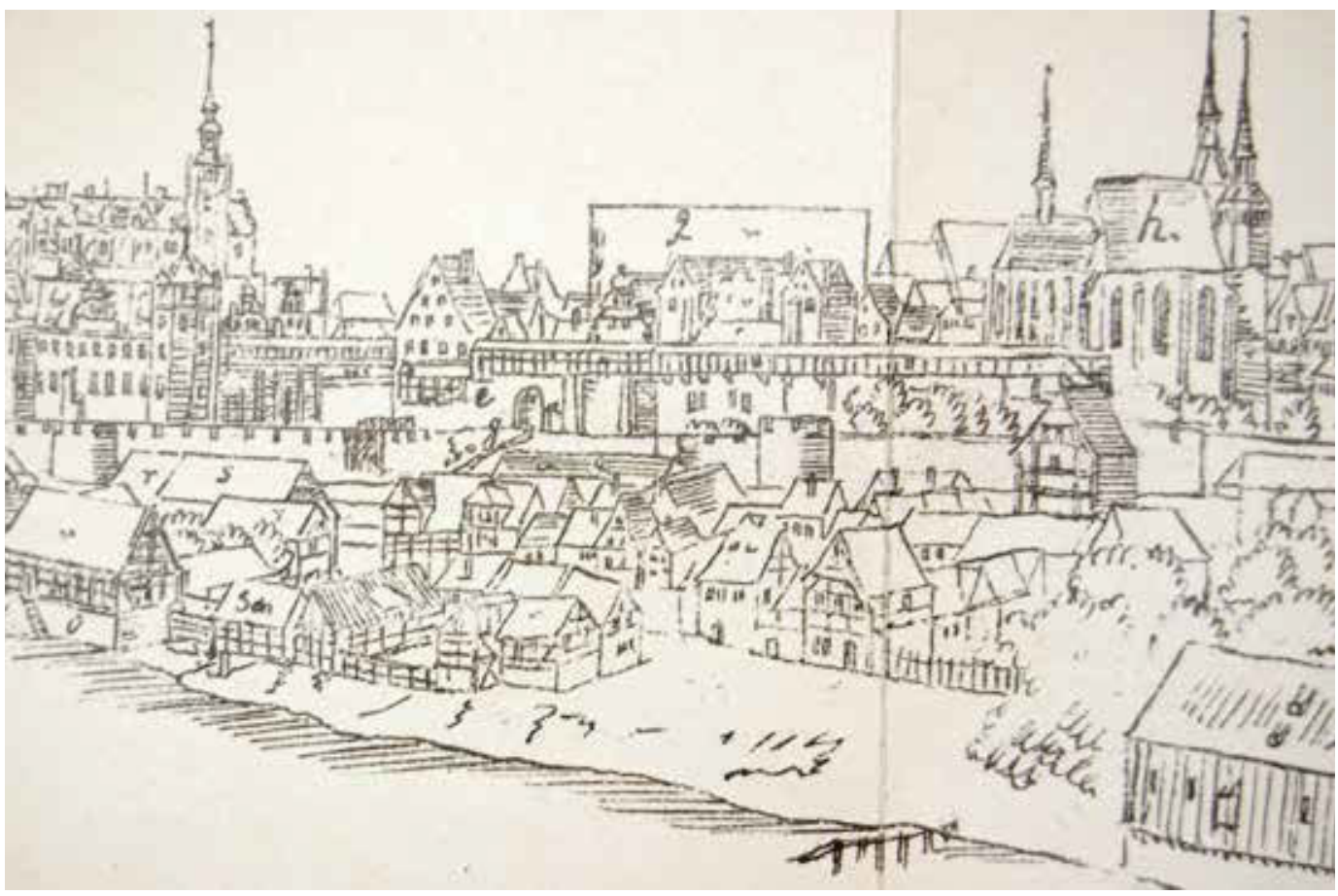


dass die Vergebung aller Schuld um des Opfers Jesus Christus Willen und aus göttlicher Gnade erfolge und nicht für gute Werke, Spenden, Ablässe und Privatmessen zu haben sei, allgemein in der Bürgerschaft Torgaus durchzusetzen. Zunächst richtete sich der Unwillen gegen die auswärtigen Bettelmönche, die Terminierer des Leipziger Paulinerklosters, des Herzberger Augustinerklosters und die Serviten aus Großenhain. Sie wurden seit 1521 durch den Rat und die Bürgerschaft bedrängt, schließlich zum Verkauf ihrer Termineigebäude und zum Verlassen der Stadt gezwungen. ${ }^{12}$

Seit 1522 musste sich der Torgauer Rat laufend mit den sich schnell verändernden Verhältnissen auseinandersetzen. So im Januar mit der Entlohnung eines namentlich nicht genannten reformatorischen Predigers an der Nikolaikirche. Sie sollte von den Bruderschaften und Handwerkern aufgebracht werden. ${ }^{13}$

Wegen mangelnder Spendenbereitschaft zur Versorgung der Stadtarmen sah sich der Rat gezwungen, einen „gemayn kasten“ einzuführen, der erstmalig am 15. Januar 1522 erwähnt wird. ${ }^{14}$ Am darauffolgenden 22. Januar beschloss der Rat darüber hinaus, um „[...] gemaynes nutzs willen ein Kasten zu vnsre lieben frawen in die Kirche desgleichen eynen zu sant Nicl(as) zusetzen [...]“..15

Die Befürchtungen einer Radikalisierung werden Anlass gewesen sein, zwei Verantwortliche $\mathrm{zu}$ bestimmen ,[...] buchsen vnd zeuck in wirden $\mathrm{zu}$ halden vnd was noth zumachen lassen auff das man in notdorfft mocht geschickt seyn $[\ldots]$ ".. ${ }^{16}$

Am 16. März 1522 protokollierte der Rat, dass man diejenigen, die während der Fastenzeit Fleisch gegessen hatten, bestrafen solle: „[...] Desgleichen die Jhenigen so bilde aus den Kirchen aus eym freveln nehmen vnd tragen verbrennen sol man vorkomen, nicht dulden vnd die verbrecher in straff nehmen [...].“ Da man sich über die Bestrafung der Verfehlungen während der Fastenzeiten nicht einigen konnte, mussten die Übertreter lediglich versichern, den Rat schadlos zu halten, wenn er vom Landesherrn „angefochten“ würde. ${ }^{17}$

Nachdem Herzog Georg von Sachsen das Reichsregiment veranlasst hatte, gegen die reformatorischen Neuerungen einzuschreiten, entschloss sich der Meißner Bischof Johann VII. im Februar 1522 zu einer Visitationsreise, um gegen die „Zerrüttung und Umstoßung der Ordnung der heiligen christlichen Kirche“ vorzugehen. Am 4. April abends traf der Bischof mit 29 Pferden in Begleitung des Leipziger Theologieprofessors Hieronymus Dungersheim und des für den Archidiakonatsbezirk zuständigen Dechanten D. Johann Hennig in Torgau ein. ${ }^{18}$ Er nächtigte bei dem zum Torgauer Stadtadel gehörigen Wolf von Dommitzsch in der Leipzigergasse (heute Leipziger Straße 5). Man trank gemeinsam ein Fass Torgauer Bier, ein weiteres kaufte der Bischof. ${ }^{19}$ Am Folgetag predigte zunächst der Bischof und nach ihm der Dechant Hennig. Hennig schloss seine Predigt mit der Ermahnung, es ist „[...] der Kirche mehr zu glauben, den dem wort Christi vnd evangelio [...] Itzund aber seint leider vil menschen, die wollen nit fasten, die nit beten, die nicht beichten, die nit under einer gestalt das sacrament entpfahen etc. vnd nichts thun, das die Kirche geordnet hat. Aber es wirt dich gereuen vnd, so du kontest, woltestu die ganze werlt darumb gebn, das du bei der Kirchen werst bliben. Darum bit ich, wollet im glauben vnd gehorsam der Kirchen bleiben." Nachmittags erfolgte ein Verhör des reformatorischen Torgauer Predigers durch den Bischof und Dungersheim. ${ }^{20}$ Es scheint sich bei dem Prediger um Valentin Tham gehandelt zu haben und für ihn zu einem Predigtverbot gekommen zu sein, da er in den unmittelbar darauf folgenden Jahren nicht mehr im Kirchendienst stand.

Dass jetzt auch der Torgauer Rat von altgläubigen Positionen abzuweichen begann, ergibt sich aus einem Beschluss vom 7. April 1522, nach dem entschieden wurde, die Katharinenkirche $\mathrm{zu}$ verkaufen und mit dem Geld ein Siechenhaus im Hospital zum Heiligen Geist zu bauen. Andererseits beschloss er, die Karfreitagsprozession ,[...] mit den figuren ditzmals [...]“ noch bestehen zu lassen. ${ }^{21}$ Der Verkauf der Katharinenkirche lag in der alleinigen Verfügungsgewalt des Rates, da sie von der Ratsbruderschaft gestiftet und errichtet worden war und um die Weihe im Mai 1514 gebeten wurde. ${ }^{22}$ Auch zwei Ratsspenden für Martin Luther im Jahr 1522 bestätigen eine veränderte Haltung, eine wurde für „Zehrung“ auf seiner Durchreise nach Herzberg gewährt, die zweite dürfte im Zusammenhang mit seinem Torgauer Predigtaufenthalt am 6. Mai 1522 stehen, wofür ihm ein Fass Bier nach Wittenberg geschickt worden ist. ${ }^{23}$ Dieser Predigtaufenthalt Luthers wurde von der kurfürstlichen Administration durch Boten auch in den Nachbarstädten Belgern, Dommitzsch und Schildau angekündigt. ${ }^{24}$ Hier kann es sich nur darum gehandelt haben, für eine allgemeine Beruhigung nach den Wittenberger Unruhen zu sorgen.

Am 9. Mai 1522 mussten sich die Räte auf Forderung der Vertreter der Gemeinde, der Hauptleute (Viertelsmeister), erneut mit der unzureichenden Entlohnung des reformatorischen
12 Herzog, Kirche und Reformation (wie Anm. 4), S. 165166.

13 StAT H 667, Ratsprotokolle 1522, 4 r.

14 Carl Gottfried Niese, Urkunden, Briefe, Exzerpte zur Torgauer Geschichte, Schriften des Torgauer Geschichtsvereins Bd. 7, Hrsg. Jürgen Herzog, Beucha/Markkleeberg 2014, S. 91, Nr. 53.

15 StAT H 667, Ratsprotokolle $1522,5 \mathrm{v}$.

16 Ebd. $7 \mathrm{v}$.

17 Ebd. 11r und StAT H 669, Ratsprotokolle 1523, 8 r.

18 Karl Pallas, Die Visitationsreise des Bischofs Johann VII von Meißen im Kurfürstentum Sachsen, in: Zeitschrift des Vereins für Kirchengeschichte in der Provinz Sachsen, VI. Jahrgang, Magdeburg 1909, S. 40 und 42, danach zitiert.

19 Jürgen Herzog, Braurechte und Bierproduktion am Anfang des 16. Jahrhunderts in der Stadt Torgau, in: Neues Archiv für Sächsische Geschichte 84 (2013), S. 34.

20 Karl Pallas, Briefe und Akten zur Visitationsreise des Bischofs Johannes VII. von Meißen im Kurfürstentum Sachsen 1522, in: Archiv für Reformationsgeschichte 19 (1908), S. 232 f, $271-274$ (Predigten), 275 - 278 (Verhör), danach zitiert.

21 StAT H 667, Ratsprotokolle $1522,13 \mathrm{v}$.

22 Ebd. H 660, Ratsprotokolle $1514,18 \mathrm{r}$.

23 Thüringisches Hauptstaatsarchiv Weimar, Ernestinisches Gesamtarchiv (im Folgenden: THStAW-EGA), Reg. Bb 3057, Ratsrechnung Torgau 1522, 31v.

24 Georg Buchwald, Lutherana, in: Archiv für Reformationsgeschichte 25 (1928), S. 2.

25 StAT H 667, Ratsprotokolle 1522, 16r. 
26 Anne-Katrin Köhler, Geschichte des Klosters Nimbschen, Leipzig 2003, S. 117 f. Eine Immatrikulation in Leipzig ist in den Matrikeln jedoch nicht feststellbar.

27 Karl-Heinz Lange, Häuserbuch der Stadt Torgau, Leipzig 2013, S. 69; THStAWEGA, Reg. Pp 3502, 1-17, Türkensteuerregister der Stadt Torgau 1531, 11r.

28 Köhler, Kloster Nimbschen (wie Anm. 26), S. 117.

29 THStAW-EGA, Kopialbuch 14, II. Abt., 13r-14r

30 Ludwig SCHMIDT, Hrsg. Urkundenbuch der Stadt Grimma und des Klosters Nimbschen, Leipzig 1895, S. 336 f., Nr. 476.

31 Martin Treu, Martin Luther und Torgau, Wittenberg 1995, S. 17, dort falsch datiert.

32 StAT, B 897, danach zitiert.

33 WA BR 3, S. 534, Nr. 894 und S. 539 , Nr. 898.

34 THStAW-EGA, Reg. B 1035, Klosterflucht Sitzenroda, $13 r$ - 16r; Weiteres zum Gesamtvorgang bei Jürgen Herzog, Klosterflucht aus Sitzenroda im Jahr 1523, in: Beiträge zur Residenz- und Reformationsgeschichte der Stadt Torgau, Schriften des Torgauer Geschichtsvereins, Bd. 8, Beucha/Markkleeberg 2015, S. 89-102.

35 StAT U 192, 232v.

36 Ebd. H 669, Ratsprotokolle 1523, 10r.

37 Ebd.
Predigers auseinandersetzen und beschlossen, seine Entlohnung zu sichern. ${ }^{25}$

Neben Johann Walter (1496-1570), dem Begründer der evangelischen Kirchenmusik und des bürgerlichen Kantoreiwesens, dürfte heute der umtriebige Kaufmann Leonhard Köppe (1464-1552) der bekannteste Torgauer Bürger der Reformationszeit als Fluchthelfer von neun Nonnen aus dem Kloster Nimbschen, unter denen sich Katharina von Bora (1499-1552), die spätere Frau Martin Luthers befand, sein. Aus einer wohlhabenden Bürgerfamilie stammend, soll er 1495 die Universität Leipzig und 1497 Erfurt besucht haben. ${ }^{26}$ Als Besitzer eines großen brauberechtigten Grundstücks in der Leipzigergasse (heute Leipziger Straße 14) versteuerte er 15311.500 Gulden einschließlich 350 Gulden Handelskapital. ${ }^{27}$ Köppe war frühkapitalistischer Unternehmer, betrieb Bierproduktion, handelte im Gewandschnitt, mit Eisen, Glas und Fisch. Das Kloster Nimbschen bezog von ihm mit klostereigenen Fuhrwerken Bier und Fisch. ${ }^{28}$ Sein aufwendigstes Vorhaben dürfte der Betrieb einer Glashütte in der Torgauer Heide gewesen sein. ${ }^{29}$

Der Fluchtvorgang ist im Einzelnen nicht überliefert, was zur Legendenbildung beigetragen hat. Lediglich vom Vaterabt des Klosters Nimbschen, Petrus von Pforta, liegt eine Beschwerde an Kurfürst Friedrich vor, nachdem „[...] nehst an dem heiligen ostertage [...]“ neun Nonnen aus dem Kloster entwichen sind. Darüber hinaus hätten einige vom Adel und ihre Verwandtschaft am Pfingstmontag weitere drei Nonnen gegen den Willen der Äbtissin aus dem Kloster

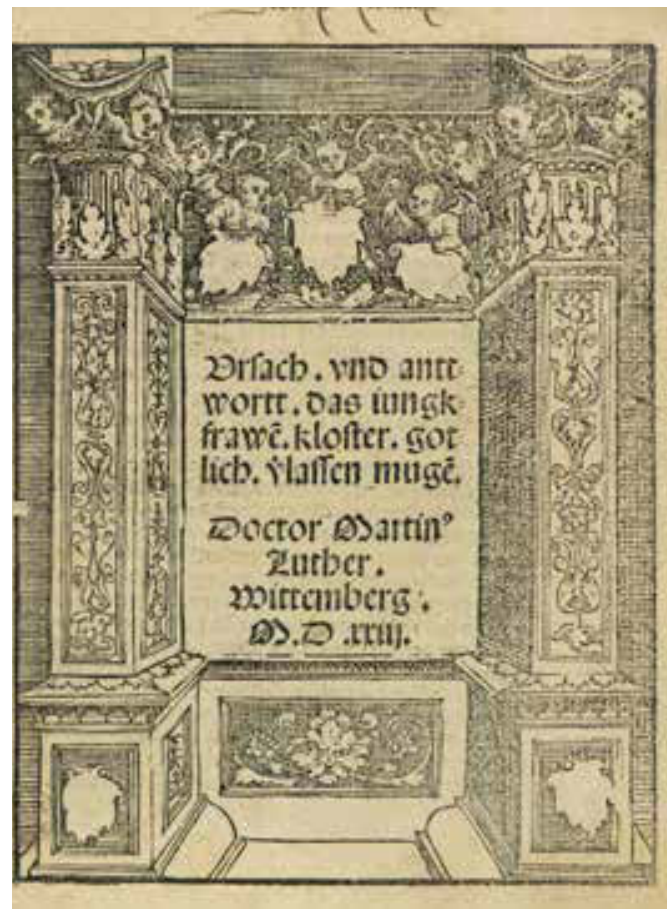

genommen. Der Abt forderte keine Rückkehr der Entwichenen, wohl aber, derartiges künftig zu verhindern.

Der Kurfürst reagierte nicht, außer festzustellen, dass er sich ,[...] dergleichen sachen nie angenomen [...]“.30 Die flüchtigen neun Nonnen werden am Ostertag, dem 5. April 1523, in Torgau angekommen und zwei Tage später nach Wittenberg gebracht worden sein, wo sich Luther um ihre Unterbringung und Versorgung bemühte. ${ }^{31}$

Luther übernahm in einem gedruckten Sendschreiben „Ursach vnd anttwort das iungkfrawen kloster gotlich v(er)lassen mugen“ die Verantwortung für diese Klosterflucht und rechtfertigte Köppes Verhalten. ${ }^{32}$ Fortan bestand eine enge Freundschaft zwischen Luther und Köppe, so dass Köppe und seine Frau auch zur Hochzeit des Reformators 1525 geladen worden sind. ${ }^{33}$

Eine weit weniger bekannte Fluchthilfe Köppes ist gut dokumentiert. In der Nacht vom 21. zum 22. Mai 1523 verhalf er nochmals fünf Nonnen zur Flucht aus dem Benediktinerinnenkloster Sitzenroda. In einem Rechtfertigungsschreiben vom 20. August erklärten er und der Mitbeteiligte Wolf von Dommitzsch: „[...] dye Junckfrawen gedencken nach Christlicher Ordnung In Ehestandt den wir fur den wurdigsten Orden schazenn zu tretten, wie derselbenn eins teyls gethann, dem tewfflischen müssigk gehenn von dem bisher alle laster entstanndenn wie am tage des Alle gestifft vnnd Closter voll sein zu entflyhenn vnd sich [...] Ehrlich Nehren mugen [...].“ Auch hier reagierte Kurfürst Friedrich der Weise nach seinem Grundsatz, dass ihn die geistlichen Dinge nichts angingen, nicht weiter. ${ }^{34}$ Das Jahr 1523 war nicht nur ein Jahr der Unruhe, sondern auch das der Abkehr der Bürgerschaft von der altgläubigen Geistlichkeit und von Spendenbereitschaft. Junge Ziegeldecker und Messerschmiede veranstalteten zur Fastnacht mit einem Kreuz eine Spottprozession und haben ,[...] schamparlid gesungen das Creuz wider die pfar gewurffen [...]“.35 Der Rat beschloss zur besseren Aufsicht über die Stadt am 25. Juni 1523, zwischen den beiden Türmen der Nikolaikirche einen offenen Gang zu errichten „[...] dormit der Hawsma(n) oder Wecht(er) allenthalb vber die stad sehen moge vnd wachen“. ${ }^{36}$ Am selben Tag mussten sich die Torgauer Räte auf Befehl des Kurfürsten erneut mit der Entlohnung des reformatorischen Predigers auseinandersetzen. Sie beschlossen, „das der ytzige $\mathrm{p}(\mathrm{re})$ diger noch eyn Jhar alhier bleibt vnd wort gots dem volg furtregt [...]“. Die Entlohnung sollte in Höhe von 20 Gulden aus dem Einkommen der Bruderschaften und anderem 
Ausbringen erfolgen. ${ }^{37}$ Es kann sich bei dem namentlich hier nicht genannten Prediger nur um den Magister Balthasar Arnold (1470-1565) handeln, der erstmalig in Torgau im Juli 1523 als ,[...] der wirdige Achtbare vnd vorsichtig(e) [...] $\mathrm{p}\left(\right.$ re)diger $[\ldots]$ “. genannt wird. ${ }^{38} \mathrm{Im}$ Jahr 1470 geboren, wird er am fürstlichen Hof in Torgau 1510 und 1511 als Priester erwähnt. ${ }^{39}$ 1519 immatrikulierte er sich an der Wittenberger Universität und promovierte dort 1521 zum Magister. ${ }^{40}$ Er gehörte zu den beliebtesten und gemäßigten Geistlichen der Stadt und starb in hohem Ansehen im Alter von 95 Jahren. ${ }^{41}$

Jetzt wurden nach dem Verkauf der Katharinenkirche die drei Hospitäler, das zum Heiligen Geist, das der Jakobsbruderschaft und das Katharinenhospital, unter Aufsicht des Bürgermeisters Erasmus Köppe, des Ratsherrn Merten Lamp und des Spitalmeisters Hieronymus Niemann zusammengeführt. ${ }^{42}$ Lediglich über das Georgenhospital, womit der altgläubige Pfarrer belehnt war, konnte der Rat noch nicht verfügen. ${ }^{43}$ Am 8. August 1523 predigte Martin Luther erneut in der Stadt, wohl wieder in der Nikolaikirche. Er erhielt vom Rat ein Weingeschenk für drei Groschen sieben Pfennig. ${ }^{44}$ In der Kirche Unser lieben Frauen und in der Franziskanerklosterkirche wurden noch immer altgläubige Messen und Gottesdienste bis 1525 durchgeführt.

Das veränderte Spendenverhalten der Bürgerschaft wird aus der Anzahl der Bierspenden durch Gott für Klöster, Termineien, Seelmessen und die Schuhknechtsbruderschaft erkennbar. Waren es bis zum Tranksteuerrechnungsjahr 1521/1522 jährlich etwa 90 Fass, so wurden 1523/1524 nur noch zwei Fass Bier gespendet. ${ }^{45}$ Am 13. Dezember 1523 predigte in der Hospitalkirche vor der Stadt erstmals der Magister Gabriel Zwilling (1487-1558), genannt Didymus, ein Mann, der die nächsten Jahrzehnte der Reformation in Torgau prägen sollte. ${ }^{46}$ Wer ihn nach Torgau berufen hat, ist nicht geklärt. Didymus hatte bereits eine bewegte Vergangenheit hinter sich. Wie Luther Mitglied des Augustinerordens, immatrikulierte er sich 1512 an der Universität Wittenberg und erwarb dort 1519 den Magistergrad. ${ }^{47}$ Zum Neujahrstag 1522 predigte er in Eilenburg und reichte das Abendmahl in beiderlei Gestalt. Danach kam es dort zu Tumulten und zur Erstürmung der Pfarre..$^{48}$ Am 10. Januar 1522 war er wieder in Wittenberg, wo er mit weiteren Mönchen im Hof des Augustinerklosters das Inventar der Klosterkirche - hölzerne Bild- und Schnitzwerke, Kruzifixe und anderes - verbrannte und steinerne Bildwerke zertrümmerte. ${ }^{49}$

Nach der Rückkehr Martin Luthers Anfang März 1522 von der Wartburg nach Wittenberg

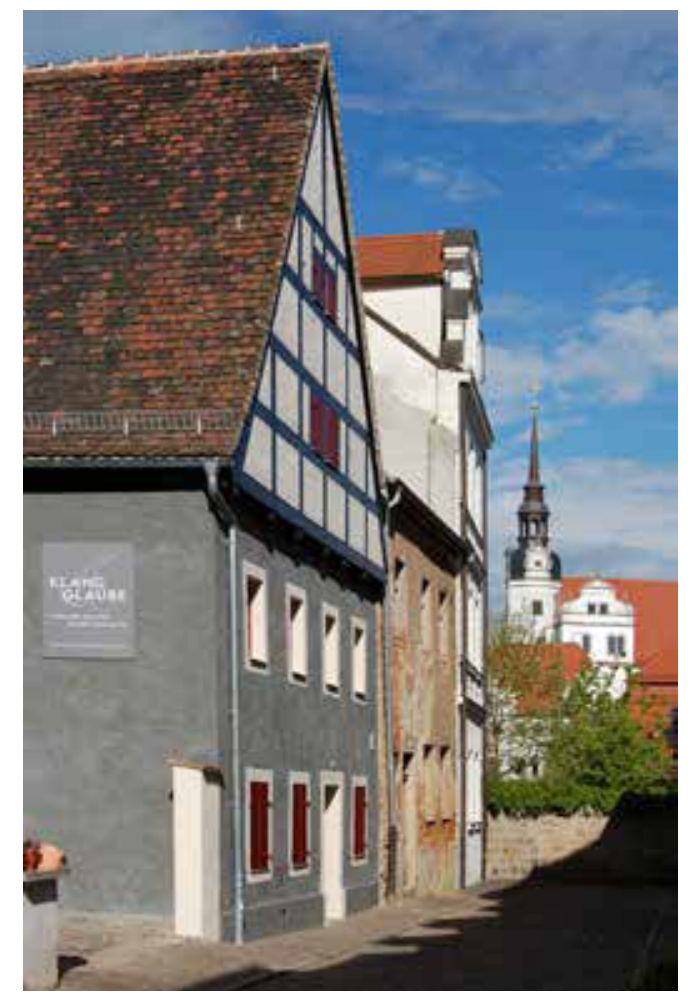

Haus des Altarlehens zum Neuen Heiligen Kreuz (Spalatinhaus), 2017

๑) Jürgen Herzog

38 StAT H 668, Ratsbuch 1523 $1528,10 \mathrm{r}$

39 THStAW-EGA, Reg. Bb 2413, Amtsrechnung Torgau $1510 / 1511,85 \mathrm{v}$ und $87 \mathrm{r}$; Adolf Aber, Die Pflege der Musik unter den Wettinern und wettinischen Ernestinern, Bückeburg/Leipzig 1921, S. 43.

40 Karl Eduard Förstemann, Album Akademiae Vitebergensis, Bd. 1, Leipzig 1841, Spalte 2, Zeile 24; Julius Köstlin, Die Baccalaurei und Magistri der Wittenberger Philosophischen Facultät 1518 1537, Halle 1888, S. 18.

41 StAT H 47, Wilhelm Krudthoff, Sammlung allerley alter Torgauischen Begebenheiten, 1754, S. 73.

ordnete er sich dem Reformator unter und genoss dauerhaft seinen Schutz. Luther empfahl ihn als Prediger für Altenburg. ${ }^{50}$ Unter dem Einfluss des altgläubigen Altenburger Augustinerchorherrenstifts und des Kurfürsten wurde Didymus aber dort nicht geduldet und musste die Stadt wieder verlassen. ${ }^{51}$

Noch 1523 erfolgte die Belehnung des kurfürstlichen Geheimsekretärs und Hofpredigers, des engen Freundes Martin Luthers, Georg Spalatin (1484-1545), mit dem Altarlehen zum Neuen Heiligen Kreuz in der Kirche Unser lieben Frauen. Das Lehen war mit jährlich 62 Gulden dotiert, davon standen dem belehnten Priester 50 Gulden zur Verfügung, die Spalatin bis zu seinem Lebensende erhalten hat. Dass er die wöchentlich zu haltenden zwei gesungenen und drei gelesenen Messen noch hat durchführen lassen, erscheint zweifelhaft, erklärte er doch im selben Jahr, sich vom Papsttum getrennt zu haben.

Das zum Lehen gehörige Priesterhaus im Sack (heute Katharinenstraße 8) bewohnte Spalatin bis zur Übernahme der Pfarrstelle in Altenburg 1525. 1526 war es an den Organisten und Mädchenschulmeister Andreas Hofer vermietet, der dort auch den Schulunterricht durchführte. Die Belehnung Spalatins ist als eine Einkommenssicherung für ihn anzusehen. Das Haus wurde ihm 1525 geschenkweise von Kurfürst Johann erblich überlassen und 1533 von ihm verkauft. Damit wurden Einkommen und Haus entgegen der Visitationsordnung von 1529 dem Gemeinen Kasten mit Bewilligung Martin Luthers entzogen. ${ }^{52}$
42 StAT H 669, Ratsprotokolle 1523, 10v.

43 Niese, Urkunden (wie Anm. 14), S. 105 f, Nr. 66

44 THStAW-EGA, Reg. Bb 3058, Ratsrechnung Torgau 1523, S. 27.

45 Herzog, Braurechte (wie Anm. 19), S. 35

46 Johann Theodor Lingke, Nachrichten von der im letzten Kriege zu einem Magazin gebrauchten Closterkirche zu Torgau ..., Torgau 1764, S. 9.

47 Fritz Bürger und Gottfried Wentz, Germania Sacra, Bd. 3, Berlin 1941, S. 484.

48 Karl Pallas, Der Reformationsversuch des Gabriel Didymus in Eilenburg und seine Folgen 1522-1525, in: Archiv für Reformationsgeschichte 9 (1912), S. 351 und 356.

49 Stefan Oehmig, Die Wittenberger Bewegung 1521/22, in: 700 Jahre Wittenberg, Weimar 1995, S. $122 \mathrm{f}$.

50 WA BR 2, S. 505, Nr. 477.

51 Hans Joachim Kessler, Altenburg zur Zeit Spalatins (1511 - 1545), in: Georg Spalatin, Hrsg. Armin Kohnle, Christine Meckelnborg und Uwe Schirmer, Halle 2014, S. 212 f.

52 Jürgen Herzog, Georg Spalatin und sein Torgauer Altarlehen, in: Schriften des Torgauer Geschichtsvereins, Bd. 8 (wie Anm. 34), S. 75-87. 
53 StAT H 670, Ratsprotokolle 1524, 1r und 3r-6v.

54 Lange, Häuserbuch (wie Anm. 27), S. 41.

55 StAT H 670, Ratsprotokolle $1524,9 \mathrm{v} / 1$

56 THStAW-EGA, Reg. Bb 3059, Ratsrechnung 1524, $28 \mathrm{v}$.

57 Germania Sacra (wie Anm. 47), S. 485.

58 StAT H 670, Ratsprotokolle $1524,18 \mathrm{v}-22 \mathrm{r}$.

59 Krudthoff, Stromateus (wie Anm. 7), S. 118.

60 StAT H 670, Ratsprotokolle $1524,28 \mathrm{v}-30 \mathrm{r}$.

61 Ebd. 9v, 11v, 17r

62 THStAW-EGA, Reg. Bb 3059, Ratsrechnung 1524, 25r.

63 Carl Knabe, Die Torgauer Visitations-Ordnung von 1529, Torgau 1881, S. 13-14.

64 Ferdinand Doelle, Der Klostersturm von Torgau im Jahr 1525, in: Franziskanische Studien, Beiheft 14, Münster. i. W. 1931, Beilage 2, S. 87, danach zitiert.

65 Ebd. S. 31-40.

66 Ebd. S. 102, Beilage 12r.

67 Lingke, Closterkirche (wie Anm. 46), S. 11, Anm. 13.

68 StAT H 670a, 1525, 7v-11v,

69 Ebd. $13 v-14 v$.
Heute dient das 1493/1494 errichtete Priesterhaus einer musealen Ausstellung zu Georg Spalatin und Johann Walter, dem Begründer der evangelischen Kirchenmusik und des bürgerlichen Kantoreiwesens.

Auch noch im Jahr 1524 versuchte der Rat, Konflikte zu vermeiden und sowohl der alten Geistlichkeit als auch den Forderungen der Gemeinde gerecht zu werden. Als am 11. Januar die jährlich übliche Versammlung der Bürgerschaft auf dem Rathaus zur Verkündung des neuen Rats und $\mathrm{zu}$ Vorhaltungen und Ermahnungen stattfand, forderte Bürgermeister Erasmus Köppe an erster Stelle, dem altgläubigen Pfarrherrn Thomas Moller die Opferpfennige „[...] wie vor alters gewest [...]“ in die Kirche zu bringen, darüber hinaus unter anderem Gotteslästerung und Nachtgeschrei zu unterlassen und für die Armen im Spital zu spenden. Die Gemeinde forderte hingegen in ihrem üblichen anschließenden Einbringen, die beiden Prediger Balthasar Arnold und Gabriel Didymus auch dieses Jahr zu behalten. ${ }^{53}$ Der Rat folgte der Aufforderung der Gemeinde und verständigte sich mit dem alten Pfarrherrn, der ohnehin gegen die reformatorischen Veränderungen nicht in Erscheinung getreten war. Balthasar Arnold gab den Kirchendienst auf und heiratete im selben Jahr die wohlhabende Witwe des 1523 verstorbenen kurfürstlichen Sekretärs Hieronymus Rudelauf und wurde damit Bürger der Stadt. ${ }^{54}$ Möglicherweise wollte er nichts mit dem radikal gesinnten Didymus zu tun haben. Gabriel Didymus wurde dem altgläubigen Pfarrherrn als Prediger zugeordnet und vereinbart, ihn dort mit Kost und „notdurfft“ zu versorgen. Freigestellt wurde ihm, mit auf dem Pfarrhof zu wohnen oder ein frei gewordenes Priesterhaus zu beziehen. ${ }^{55}$ Damit trat er an die Stelle von Balthasar Arnold und scheint das Priesterhaus gewählt zu haben. Auch er heiratete 1524, wozu ihm und der Braut Fische und Wein vom Rat geschenkt worden sind. ${ }^{56}$ Die Braut soll die Witwe eines Kanzelisten Hieronymus gewesen sein. ${ }^{57}$ Am 27. April 1524 wurde die Gemeinde erneut auf das Rathaus geladen und ihr jetzt vorgehalten: „[...] Die tempel vnd Kirchen vnehret man, stilt alles daraus an vorhenge vnd tuchern der altar, Auch wirt das stelen sonst gemayn, im gart(en) holtz reis, auch sunderlich fisch [...] dodurch der Stad ein loßgericht entstehet [...] und Weyl dem pfarherr vnd seynst acidencia fast nydergefallen, szo sol doch eyn yeder wirt mit seym gesinde verfug(en), das im seyn opffer [...] gefalln, dan er beclagt sich seint im ytzt auf ostern schwerlich der drittayl einkommen [...]“. Beschlossen wurde darüber hinaus, dass an den Sonntagen eine Messe, wohl in der Niko- laikirche, zu halten sei „[...] vmb eyennis fremder lewth willen [...]“. Den belehnten Altaristen befahl der Rat, diese Messen der Reihe nach durchzuführen. Die Gemeinde forderte, die Tür am Friedhof vor der Klosterkirche weiter und größer zu machen, weil man da ,[...] stets p(re) digt [...]“. ${ }^{58}$ Es bleibt offen, ob es sich um Predigten reformatorischen Inhalts gehandelt hat. Möglicherweise besteht ein Zusammenhang mit der Entscheidung des Franziskanerordens, einen geeigneten Prediger nach Torgau zu schicken. Der Rat wurde davon am 24. August 1524 informiert und gebeten, „[...] solchen Vater vnd Bruder behäglich annehmen, vnd ym in christlichen sachen, gutwilligkeit erzeigen". ${ }^{99}$

Widersprüchlich erscheinen die auf Auseinandersetzungen hinweisenden Forderungen zur Gemeindeversammlung am 12. Oktober. Der Rat ermahnte die Bürger „[...] wider die Monnich oder clost(er) nichts furzunehmen noch $\mathrm{zu}$ yn nicht $\mathrm{zu}$ notigen, wider an schrencken (Einfriedungen) oder andern abzubrechen bey vermeydung des rats straff [...]“. Die Gemeinde forderte hingegen: „[...] Man solle die Monniche auch $\mathrm{p}$ (re)digen lassen, so sie das Evangelium $\mathrm{p}(\mathrm{re})$ digen $[\ldots]^{\circ .}{ }^{60}$

Das Jahr 1524 war auch das Jahr der Auflösung der letzten religiösen Bruderschaften. Nachdem die Jakobsbruderschaft ihren Besitz bereits dem Rat übertragen hatte, folgten jetzt die Ratsbruderschaft, die der Kramer, Schützen und Ackerknechte. ${ }^{61}$ Im selben Jahr wurden die ersten Altarlehen durch Tod und Entschädigung der Inhaber vom Gemeinen Kasten eingezogen die Lehen Maria Magdalena ${ }^{62}$, Hieronymi und Pauli der Kirche Unser lieben Frauen und Crispini und Christiani der Nikolaikirche. ${ }^{63}$

Ein Ereignis, dass den höchsten Unmut des Kurfürsten Friedrich des Weisen in seinen letzten Lebenswochen nach sich zog und das bisherige wohlwollende Verhältnis zum Torgauer Rat in Frage stellte, war der Sturm des Franziskanerklosters in der Nacht vom 28. Februar zum 1. März 1525 durch 24 Bürger und Bürgersöhne, nachdem sie dem Fastnachtsbier zugesprochen hatten. Nach dem Bericht der geflohenen Anführer, des Hofschneiders Günther Braun und des kurfürstlichen Büchsenmeisters Paul Resch, habe der franziskanische Kustos von Meißen D. Jakob Schwederich bei einem Essen im Haus des Bürgermeisters Erasmus Köppe und in Gegenwart von Gabriel Didymus Zweifel an der Wahrhaftigkeit der Evangelien geäußert. Wegen dieser ,[...] erschrecklicher rede [hat] der prediger vffentlich vor allem volck auff der canzeln beclaget vnd gesagt, eynen sulchen, der eyn crist gerwmett seyn will vnd solche wort, das das heilige ware evangelium nicht von Gott, 
nach aus seinen gotlichen munde beschrieben were, von jhm lawten ließe, solt man zwr stadt mit ruthen hinaus hawen [...] vnd wir armen gesellen dardurch ane zweyvell aus vorhencknus vnd straffe des almechtigen neben andern zw solchem furnehmen vorvrsacht $\left[\ldots\right.$. ]“. ${ }^{64}$

Dem ersten Klostersturm folgte ein zweiter von unbekannt gebliebenen Tätern, der mit Plünderung verbunden war. In der Kirche ist die Tür zur Sakristei zerschlagen worden, im Chor wurden Lesepulte, Messingleuchter, Chorgestühl, Zinnleuchter und Schlaguhr beschädigt und Vorhänge zerrissen. Das Altargemälde vom Franziskusaltar wurde herabgeworfen und beschädigt. Die Mönche wurden verprügelt und ihrer Kleidung beraubt. ${ }^{65}$

Der Kurfürst verdächtigte den Torgauer Rat der Anstiftung, zumal er ausdrücklich befohlen hatte, nichts gegen die Geistlichkeit und das Kloster zu unternehmen. Seine Entrüstung wurde noch dadurch gesteigert, dass er selbst zu diesem Zeitpunkt in Torgau war und eine Gesandtschaft Erzherzog Ferdinands empfangen hatte. Sein Bruder Herzog Johann und der Kurprinz Johann Friedrich, an die sich die Klosterstürmer mit Bittgesuchen wandten, beurteilten den Vorgang wesentlich milder. ${ }^{66}$ Der Torgauer Rat beteuerte seine Unschuld, die Schäden wurden beseitigt und die Kosten in Höhe von neun Schock, 27 Groschen und sechs Pfennigen aus dem Gemeinen Kasten beglichen. ${ }^{67}$

Nach dem Tod Kurfürst Friedrich des Weisen am 5. Mai 1525 veränderte sich die Situation grundlegend. Der neue Kurfürst Johann und sein Sohn Johann Friedrich waren bereits vorbehaltlos Anhänger der lutherischen Reformation. Von einer Bestrafung der Klosterstürmer ist nichts bekannt, sie blieben wahrscheinlich unter den neuen Herrschaftsbedingungen unbehelligt.

Die mit dem Klostersturm deutlich gewordene Unsicherheit für die altgläubige Geistlichkeit wird Anlass für den Pfarrherrn gewesen sein, jetzt auch sein Lehen über das Georgenhospital aufzugeben und das Spital in Gegenwart ,[...] seins predigers [...]“ Gabriel Didymus dem Rat zu übergeben. Am 20. März 1525, ,...] hat ein sitzender [regierender] Rat Allen dreyen vnd Alden Rethen fürgehald(en) wie das der Erwirdig Achtbar vnnd Hochgelerte her Thomas Molre, Doctor vnnd pfarher alhier, das spittalh zu sant Jorgen [...] mit Kyrchenn vnd den heußern mit yren Rawmen, eym Rat vbergeben hette, dasselb eintzunehmen, zugebrauch[en] vnnd zuvorkeuffen [...]“. Die Gebäude wurden in kürzester Frist als bürgerliche Grundstücke verkauft und ein Gesamtkaufpreis von 570 Gulden erzielt. ${ }^{68}$

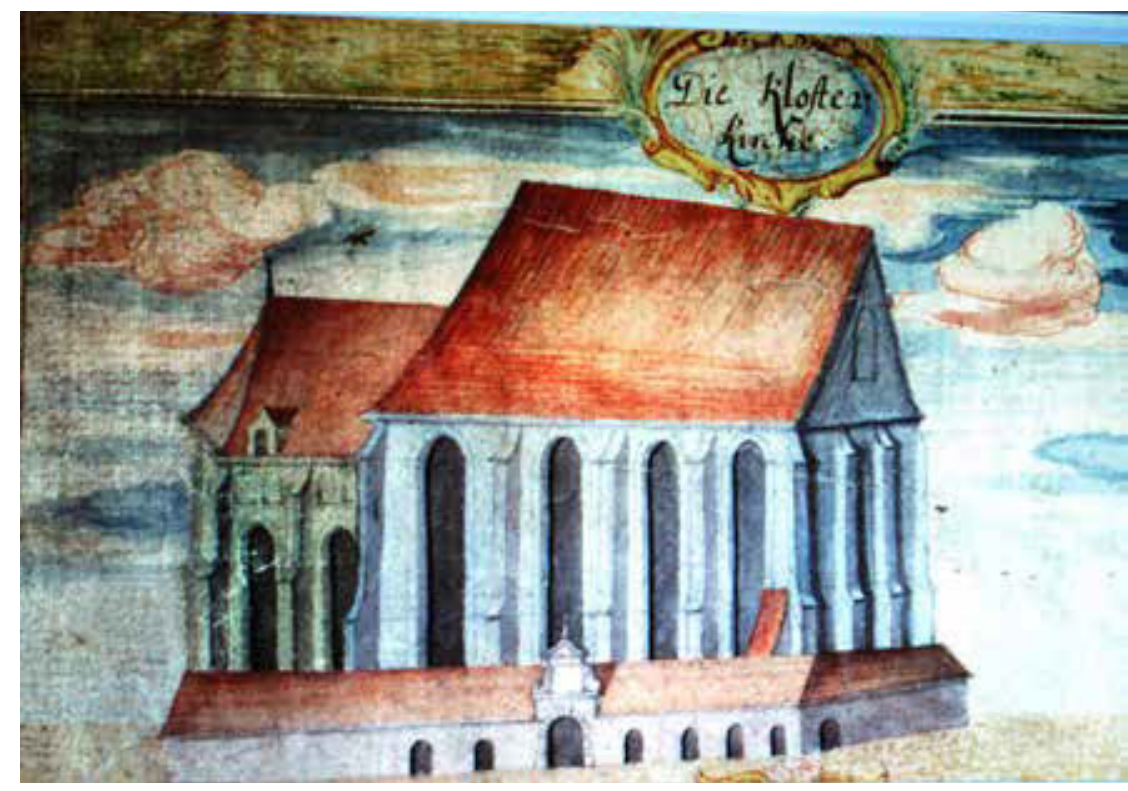

Nach dem Tod Kurfürst Friedrich des Weisen sahen sich die noch verbliebenen sechs Mönche des Franziskanerklosters des kurfürstlichen Schutzes beraubt und mussten sich schutzsuchend an den Torgauer Rat wenden. Bereits zwei Tage nach dem Tod des Kurfürsten protokollierte der Rat die Übernahme der Kleinodien und eines Hauses vor dem Kloster und sicherte den Mönchen bei ihrem Wohlverhalten Schutz zu. ${ }^{69}$ Die Kleinodien und das Haus wurden verkauft und ein Erlös von 474 Gulden dem Gemeinen Kasten zugeführt. ${ }^{70}$

Für den 17. Mai wurde die Gemeinde auf das Rathaus gerufen und durch den energischen Bürgermeister Erasmus Köppe eine Disziplinierung bei Ankündigung harter Strafen gefordert: „[...] Ebruch sol bey der Staup gestrafft werden [...] Es wirdt vil verloren vnd gestolen, sol die scherff des schwerts [...] gegen yn furgenomen, vnd die diebe geha(n)g(en) werd(en) [...]. Ein yder wie ihme geboten In bereitschaft $\mathrm{zu}$ sitz(en). Also sol er mit seiner besten Weher geschickt sein, vnd aus der stadt an erlaubung eins Rats nicht wegkzihen [...].“ Für die Franziskaner forderte er: „[...] Die Monche, wie sy vorhin ye vnd ye in des Raths schutz gewesen [...] nicht belestig(en) sundern bruderliche liebe in gedult an yn bewaysen [...]." Die Gemeinde erklärte: „[...] Dy Monche gerne zu duld(en) Alleyne sy solt(en) gleiche Kleydung mit ynen trag(en) vnd dy phariseysche ausszih(en) [...]“. Durch den Tod des alten Pfarrherrn Thomas Moller am 1. Mai 1525 war jetzt auch die Pfarrstelle vakant geworden. Die Entscheidung über die Neubesetzung überließ der Rat der Gemeinde, die sich noch während der Bürgerversammlung für Gabriel Didymus entschied. ${ }^{71}$ Schon am selben Tag bat der Rat den neuen Kurfürsten Jo-
Klosterkirche um 1720, aus: Hans Sigismund Ulrici, Amtskarte Torgau, Randzeichnung ๑ Sächsisches Staatsarchiv, Hauptstaatsarchiv Dresden, 12884 Karten und Risse, Schrank XIV, Fach IV, Nr. 8

70 Doelle, Klostersturm (wie Anm. 64), S. 63, Anm. 24.

71 StAT H 670a, Ratsprotokolle 1525, 19r-21r.

72 StAT H 38, Torgauer Chronik 1529 - 1692, S. 335-338.

73 StAT H 14, Geschichtliche Nachrichten von Torgau, 162r-v.

74 Wolfgang Kieswetter: Das man das lauter rain Evangelion / on menschliche zusatzunge predigen sol / Fürstlicher bevehl zu Weymar beschehen, Weimar 1525.

75 Willibald Gurlitt, Johannes Walter und die Musik der Reformationszeit, München 1933, S. $35 \mathrm{f}$

76 StAT H 576, Privilegien und Statuten, Torgau 1537, 2v-3r.

77 THStAW-EGA, Reg. Bb 2441 und 2443, Amtsrechnungen $1525 / 1526$ und 1526/1527, danach ermittelt

78 Doelle, Klostersturm (wie Anm. 64), S. 114, danach zitiert.

79 Knabe, Visitations-Ordnung (wie Anm. 63), S. 2-8

80 StAT H 2615, Unterricht von des Barfüßerklosters und anderer geistlicher Güter Einkommen, 1532, unpaginiert. 


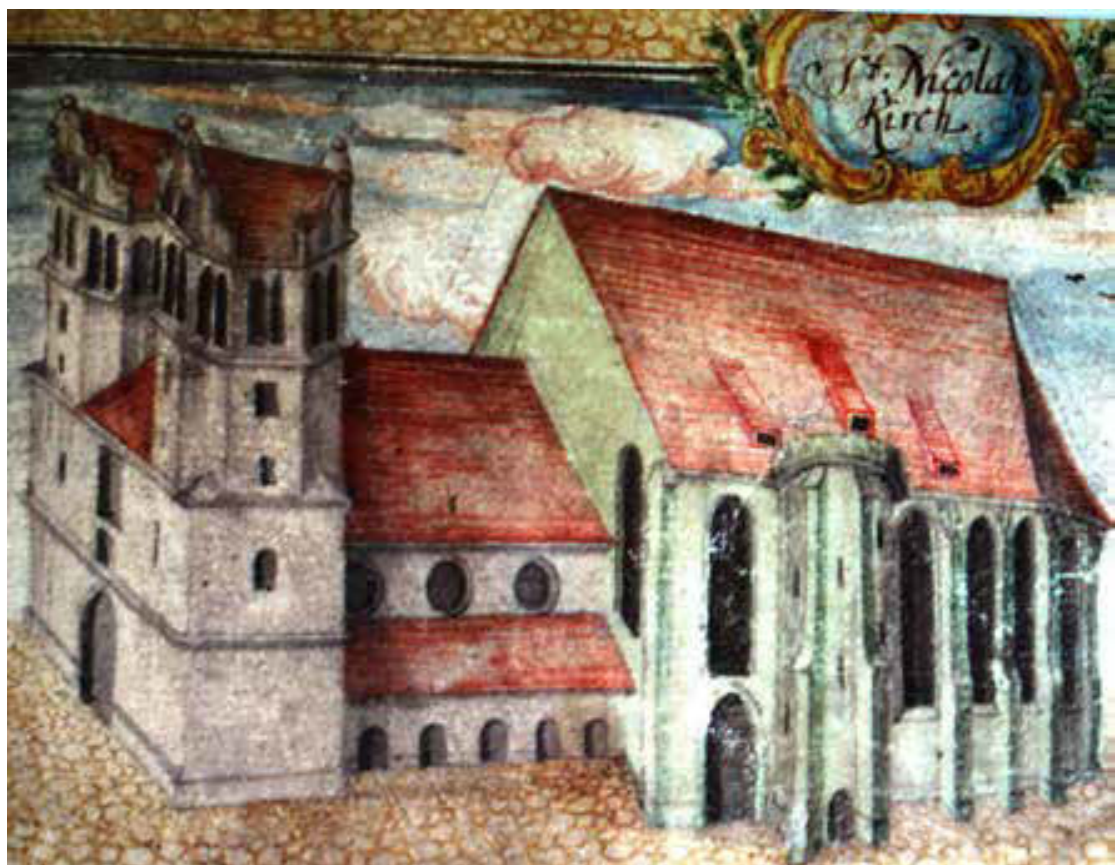

Nikolaikirche um 1720, aus: Hans Sigismund Ulrici, Amtskarte Torgau, Randzeichnung - Sächsisches Staatsarchiv, Hauptstaatsarchiv Dresden, 12884 Karten und Risse, Schrank XIV, Fach IV, Nr. 8

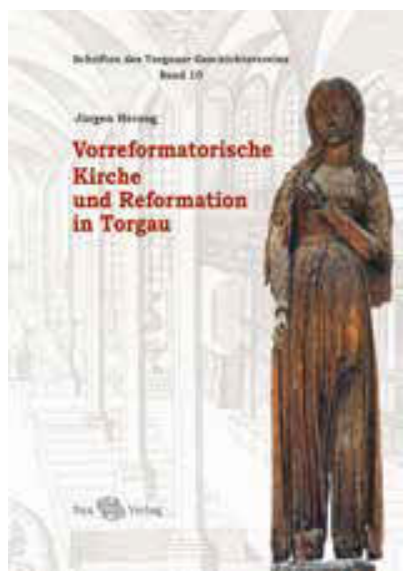

Zum Weiterlesen: Jürgen Herzog: Vorreformatorische Kirche und Reformation in

Torgau. Schriften des Torgauer Geschichtsvereins, Band 10.

Sax-Verlag Markkleeberg 2016, 632 Seiten mit zahlreichen

Abbildungen, 35,00 Euro, zu beziehen bei Sax-Verlag, Eibenweg 62, 04416 Markleeberg, Tel. 0341/3502117,info@sax-verlag.de

Autor

Dr. Jürgen Herzog Torgau hann um Zuweisung eines Pfarrherrn und schlug dafür Didymus vor. ${ }^{72}$ Die Bestätigung muss vor dem 3. Juli 1525 erfolgt sein, da Didymus von diesem Tag eine Inventaraufnahme über den Pfarrhof an den Rat übergab, den er wohl schon bezogen hatte. ${ }^{73}$

Erst nach der Niederschlagung des Bauernaufstands bereitete sich Kurfürst Johann auf den Ortswechsel seiner Hofhaltung vor. Der Priesterschaft des Amtes Weimar wurde in Weimar am 17. August 1525 vorgetragen, dass ,[...] der Churfürst / Herzog Hans vnd auch [...] Herr Johann Friedrich der junge Fürst, auß vrsach / vnd ihrer gnaden gelegenheit nach / ire Behausung / vnd hoffhaltung anderswo haben vnd halten werden $/[\ldots]$ “.. Es wurde befohlen, dass „[...] lauter rein Evangelion / on menschliche zusatzunge [...] zu predigen [...]“. Weiter wurde erklärt, dass in kurzer Zeit eine Reformationsordnung aus Wittenberg eingehen würde, die alle Zeremonien vorschreibt. ${ }^{74}$ Diese Ordnung wurde dann auch von Martin Luther unter Beteiligung des Torgauer Sängermeisters Conrad Rupsch und des Komponisten Johann Walter als Deutsche Messe erarbeitet, am 29. Oktober von Walter mit nach Torgau gebracht und dem Kurfürsten übergeben. ${ }^{75}$ Am 20. August 1525 erfolgte die Huldigung des neuen Kurfürsten in Torgau ${ }^{76}$, sein erstes Hoflager hat er vom 30. August bis zum Juni 1526 hier gehalten. ${ }^{7}$

Jetzt reichten die Schloss- und Amtsgebäude für den aufwendigen Bedarf der Hofhaltung nicht mehr aus, so dass die Gebäude des Franziskanerklosters sofort zusätzlich in Anspruch genommen wurden und das Klosterleben endgül- tig zum Erliegen kam. Der letzte Guardian des Klosters Urban Aber schrieb an den Kustos der Franziskaner in Meißen, D. Jakob Schwederich: „[...] der furste hat den convent gewaldiglichen eyngenommen [...] dy hewßer dynne zcu stallunge vnd daß gasthaw $ß$ vnde refectorium zcu harnischkammern [...] der alde vnd junge hertzog vnd das gantze hoffegesynde synt gantz erbittert vff alle monche $[\ldots]$... ${ }^{78}$

Die erste Kirchenvisitation in Torgau hat vom 20. April bis zum 10. Mai 1529 für die Stadt und den Kirchenkreis in der Trinkstube am Markt stattgefunden. Die Visitatoren, zu denen Martin Luther und Justus Jonas gehörten, fanden in der Stadt geregelte Verhältnisse vor. Geistliche und Schuldiener wurden in ihren Ämtern bestätigt, die Anzahl und der Inhalt der Predigttätigkeit vorgegeben: „[...] denn zu viel ist ungesundt, macht müde vnd überdrüssig, bede lerer vnd schüler [...]“. Für den Unterricht an der Lateinschule sind umfangreiche Vorschriften zum Umfang und Inhalt des Lehrprogramms in die Visitationsordnung aufgenommen worden.

Zum Gemeinen Kasten erklärten die Visitatoren, dass ihn ,[...] ein Radt auß gutem bedenken für uns geordnet [...]“ hat. Er war finanziell bereits gut ausgestattet, sein jährliches Einkommen wird mit 600 Gulden angegeben, weitere 648 Gulden waren zinsbar ausgeliehen.

Sanktioniert wurde, dass die Einkommen der Lehen, darunter auch der fürstlichen, nach dem Tod ihrer Inhaber in den Gemeinen Kasten fallen und die Häuser verkauft werden sollten. Geregelt wurden die Einkommensverhältnisse der Geistlichen, Schuldiener und Kastenherren.

Erstmalig ist jetzt der Pfarrer auch mit dem Superintendentenamt beauftragt worden. ${ }^{79}$

Zur Verwendung der Kirchen ist in der Ordnung nichts enthalten. Sich auf die Visitation berufend, wurde die Klosterkirche dem gottesdienstlichen Gebrauch für die Gemeinde zur Verfügung gestellt, woraus die Bezeichnung Alltagskirche gegenüber der Kirche Unser lieben Frauen als Sonntagskirche üblich geworden ist. Die Marktkirche St. Nikolai erhielt der Rat zum profanen Gebrauch, sie sei „ein aldvetterische finstere Kirche“. ${ }^{80}$ Ihr mittelalterliches Geläut blieb erhalten und läutet noch heute zweimal täglich. Das Kirchenschiff wartet noch immer auf seine Instandsetzung.

Dem Torgauer Rat ist zu danken, dass er ab 1525 sehr schnell geordnete Kirchen- und Schulverhältnisse geschaffen hat. Er besaß das Vorschlagsrecht für den Pfarrherrn und konnte die übrigen Geistlichen und die Schuldiener berufen und entlassen. Rechte, die in den folgenden Jahren zwar angefochten wurden, aber doch von Bestand blieben. 